Check for updates

Cite this: RSC Adv., 2019, 9, 35003

\title{
A dual-mode nanoparticle based on natural biomaterials for photoacoustic and magnetic resonance imaging of bone mesenchymal stem cells in vivo
}

\begin{abstract}
Hua Zhang,,$^{\mathrm{a}}$ Zhen-Jun Wang,,$^{\mathrm{bc}}$ Ling-Jie Wang, $\mathbb{D} \dagger^{\mathrm{a}}$ Ting-Ting $\mathrm{Li}^{\mathrm{b}}$ Sheng $\mathrm{He},{ }^{\mathrm{a}}$ Li-Ping Li, ${ }^{b}$ Xiao-Yan Li, ${ }^{a}$ Shi-Jie Liu, ${ }^{a}$ Jian-Ding Li, ${ }^{a}$ Si-Jin Li ${ }^{\star d}$ and Rui-Ping Zhang ${ }^{\star b c}$

Stem cell imaging in vivo is critical to elucidate the homing, distribution, survival, and repair mechanisms and to evaluate the therapeutic effects of engrafted stem cells. Unfortunately, unimodal imaging of stem cells does not simultaneously satisfy all current requirements owing to their intrinsic limitations. Obviously, bimodal or multimodal imaging of stem cells is a promising strategy for circumventing this issue. This study aimed to design and synthesize a novel dual-modal polyethylene glycol-modified magnetic nanoparticle $\left(\mathrm{Fe}^{3+}-\mathrm{PEG}-\mathrm{MNP}\right)$ based on natural biomaterials including melanin and Fe ions for photoacoustic (PA) and magnetic resonance (MR) imaging of stem cells in vivo. The $\mathrm{Fe}^{3+}-\mathrm{PEG}-\mathrm{MNPS}$ were characterized and their PA/MR imaging capability and cytotoxicity were evaluated. Bone marrow mesenchymal stem cells (BM-MSCs) labeled with $\mathrm{Fe}^{3+}$-PEG-MNPs were subjected to PA and MR imaging in vitro and in vivo. Consequently, $\mathrm{Fe}^{3+}-\mathrm{PEG}-\mathrm{MNPs}$ displayed many superior properties, including ultrasmall particle size, higher stability, water solubility, easy labeling of cells, lower cytotoxicity, high biosafety, excellent capability of PA/MR imaging, high sensitivity and long-term monitoring in vitro and in vivo. In particular, PA and MR signals of labeled BM-MSCs were maintained for at least 35 and $28 \mathrm{~d}$, respectively, in vivo. Therefore, $\mathrm{Fe}^{3+}-\mathrm{PEG}-\mathrm{MNPs}$ are ideal dual-modal PA/MR nanoparticles for noninvasive and effective monitoring of engrafted stem cells in vivo.
\end{abstract}

Received 31st July 2019

Accepted 21st October 2019

DOI: $10.1039 / \mathrm{c} 9 \mathrm{ra05937g}$

rsc.li/rsc-advances

\section{Introduction}

Stem cell therapy (SCT) has been an effective and promising regenerative therapeutic strategy for many diseases owing to its potential for multipotent differentiation and self-renewal. ${ }^{1}$ Among the numerous stem cells available, bone marrow mesenchymal stem cells (BM-MSCs) are preferred for SCT owing to minimum ethical constraints, ease of harvesting, low immunogenicity, multi-differential potentialities, and self-renewal capacity. Previous studies have reported that BM-MSC therapy is an effective alternative in various diseases, such as heart disease, cerebral infarction, renal ischemia-reperfusion injury, spinal cord injury, and hepatic failure..$^{2-6}$ However, numerous

\footnotetext{
${ }^{a}$ Department of Medical Imaging, First Hospital of Shanxi Medical University, Taiyuan, Shanxi Province 030001, China

${ }^{b}$ Department of Biochemistry and Molecular Biology, Shanxi Medical University, Taiyuan, Shanxi Province 030001, China

'The Affiliated Da Yi Hospital of Shanxi Medical University, Taiyuan, Shanxi Province 030032, China. E-mail: zrp_7142@163.com; Tel: +86-351-4639324

${ }^{d}$ Department of Nuclear Medicine, First Hospital of Shanxi Medical University, Taiyuan, Shanxi Province 030001, China. E-mail: lisjnm123@163.com; Tel: +86$351-4639278$

$\dagger$ Hua Zhang, Zhen-Jun Wang and Ling-Jie Wang are co-first authors.
}

issues regarding engrafted BM-MSCs warrant clarification, such as their migratory path in vivo, biodistribution, differentiation capacity, long-term fate, and beneficial effects. Therefore, noninvasive continuous monitoring of engrafted BM-MSCs in vivo is necessary to address the aforementioned issues.

Currently, various stem cell imaging methods in vivo have been applied for cell tracking, such as optical imaging (including fluorescence and bioluminescence imaging), magnetic resonance imaging (MRI), radionuclide imaging (including positron emission tomography (PET) and single-photon emission computed tomography (SPECT)), and photoacoustic imaging (PAI). Unfortunately, these methods have intrinsic limitations and cannot satisfy all requirements. Optical imaging methods for tracking stem cells in vivo are generally limited by their low penetration depth in tissue and phototoxicity. ${ }^{7}$ Although MRI has been widely used for cell tracking because of high tissue resolution and long-term monitoring of engrafted cells in vivo, it has relatively lower sensitivity than other methods, longer scan duration, and difficulty quantifying cell division. ${ }^{8,9}$ Despite considerable sensitivity, radionuclide imaging is restricted to the imaging of labeled stem cells in vivo owing to poor spatial resolution, a short half-life of radiotracers, and high cost. ${ }^{8,9}$ PAI not only provides high spatial resolution and sensitivity but also has a relatively greater depth of tissue 
penetration, and it is a compound imaging method of optical and ultrasound (US) imaging based on ultrasound waves induced by thermoelastic expansion of tissue after photon absorption. ${ }^{10}$ Consequently, dual-modal or multimodal cell imaging methods are important for efficient monitoring of the homing, number, and fate of engrafted stem cells in vivo.

This study aimed to develop a dual-modal photoacoustic and MR nanoprobe for stem cell imaging in vivo, comprising a nanochelate of polyethylene glycol-modified melanin and iron ions nanoparticles ( $\mathrm{Fe}^{3+}$-PEG-MNPs). We characterized $\mathrm{Fe}^{3+}$-PEGMNPs and evaluated their cellular internalization and cytotoxicity in vitro. In particular, the feasibility of PAI/MRI dual-modal long-term monitoring of $\mathrm{Fe}^{3+}$-PEG-MNPs labeled BM-MSCs in vivo was verified.

\section{Materials and methods}

\subsection{Synthesis of $\mathrm{Fe}^{3+}$-PEG-MNPs}

PEG-MNPs were obtained as described previously ${ }^{11,12}$ Briefly, 150 $\mu \mathrm{L}$ of $16 \mathrm{mg} \mathrm{mL}^{-1} \mathrm{FeCl}_{3} \cdot 6 \mathrm{H}_{2} \mathrm{O}$ aqueous solution was added into $3 \mathrm{~mL}$ of $3 \mathrm{mg} \mathrm{mL}{ }^{-1}$ MNPs aqueous solution and then stirred gently for $1 \mathrm{~h}$ at $37{ }^{\circ} \mathrm{C}$. Free $\mathrm{Fe}^{3+}$ and $\mathrm{Cl}^{-}$ions were filtered out using a PD-10 column (GE Healthcare, Chicago, IL, USA). The chelation products thus obtained were washed with ultrapure water and centrifuged at room temperature (3500 rpm, $15 \mathrm{~min}$ ) twice to further eliminate free $\mathrm{Fe}^{3+}$ and $\mathrm{Cl}^{-}$ions and then filtered through a $0.22 \mu \mathrm{m}$ membrane (Acrodisc Syringe Filters, PALL, New York, USA). The final products ( $\mathrm{Fe}^{3+}$-PEG-MNPs) were stored in sterile EP tubes for subsequent analysis.

\subsection{Characterization of $\mathrm{Fe}^{3+}$-PEG-MNPs}

The ultraviolet-visible-near infrared (UV-vis-NIR) absorption spectra of the $\mathrm{Fe}^{3+}$-PEG-MNPs were detected via ultravioletvisible-near infrared spectroscopy (UV-vis-NIR; UV-6100; MAPADA Instruments, Shanghai, China), and their morphology was observed using a transmission electron microscope (TEM; JEM-2100F; JEOL, Tokyo, Japan). The hydrodynamic diameters and zeta potential were measured via dynamic light scattering (DLS; Zetasizer Nano ZS90; Malvern Instruments, Malvern, UK).

The $\mathrm{Fe}^{3+}$ concentration in $14 \mathrm{~mL}$ of $71.55 \mu \mathrm{g} \mathrm{mL}{ }^{-1} \mathrm{Fe}^{3+}$-PEGMNPs aqueous solution was measured via inductively coupled plasma mass spectrometry (ICP-MS; ICPMS-2030; SHIMADAZU Instruments, Kyoto, Japan) to determine the concentration of chelated $\mathrm{Fe}^{3+}$ ions.

Furthermore, the stability of $\mathrm{Fe}^{3+}$-PEG-MNPs was analyzed by dissolving them in phosphate-buffered saline (PBS; $\mathrm{pH}=7.4$ ) and stirring gently at $37^{\circ} \mathrm{C}$. Free $\mathrm{Fe}^{3+}$ concentration was measured via ICP-MS at 0.5 h, 1 h, 2 h, 4 h, 8 h, 12 h, 24 h, and 48 h.

\subsection{Dual-modal PA/MR imaging of an aqueous solution of $\mathrm{Fe}^{3+}$-PEG-MNPS}

Aqueous solutions of $\mathrm{Fe}^{3+}$-PEG-MNPs and PEG-MNPs of different concentrations $(0,12.5,25,50,100,200,400$, and 800 $\mu \mathrm{g} \mathrm{mL} \mathrm{m}^{-1}$ ) were prepared for PA imaging, using a multispectral optoacoustic tomographic imaging system (MSOT) (inSight 128,
iThera Medical GmbH, Munich, Germany). Briefly, laboratoryprepared white thin plastic tubes (inner diameter of $3 \mathrm{~mm}$ ) filled with the test solution of different concentrations were placed in the phantom. After excitation with tunable pulses at 680-980 nm, photoacoustic signals of aqueous solutions of $\mathrm{Fe}^{3+}$-PEG-MNPs and PEG-MNPs of different concentrations were recorded. The PA imaging parameters were as follows: temperature, $25{ }^{\circ} \mathrm{C}$; selected positions of the phantom, 3; frames per wavelength, 4; repetitions per slice, 1; run, 1; slice thickness, $800 \mu \mathrm{m}$.

Aqueous solutions of $\mathrm{Fe}^{3+}$-PEG-MNPs and Omniscan (a contrast-enhancing agent commonly used in MRI) were imaged using a 3.0-T clinical MRI equipment (MAGNETOM Skyra; Siemens, Munich, Germany) with a wrist surface coil. The samples of $\mathrm{Fe}^{3+}$-PEG-MNPs and Omniscan aqueous solution were placed in a 96-well plate, each well containing samples of different $\mathrm{Fe}^{3+}$ and $\mathrm{Gd}^{3+}$ concentrations (0.0125, 0.025, 0.05, 0.1, $0.2,0.4$, and $0.8 \mathrm{mmol} \mathrm{L}^{-1}$ ), and deionized water was used as a negative control. The $T_{1}$ value was measured using the $T_{1}$ mapping sequence images. $T_{1} \mathrm{WI}$ parameters were as follows: field of view (FOV), $100 \times 90 \mathrm{~mm}^{2}$; slice thickness, $1.5 \mathrm{~mm}$; spacing, $0.5 \mathrm{~mm}$; base matrix resolution, $256 \times 256$; and effective echo time (TE), $20 \mathrm{~ms}$. The $T_{1}$ relaxation efficient $\left(r_{1}\right)$ values of $\mathrm{Fe}^{3+}$-PEG-MNPs and Omniscan were obtained by calculating the ratio of $1 / T_{1}\left(\mathrm{~s}^{-1}\right)$ to the $\mathrm{Fe}^{3+}$ and $\mathrm{Gd}^{3+}$ concentrations $\left(\mathrm{mmol} \mathrm{L}^{-1}\right)$.

\subsection{Labeling and assessment of the cytotoxicity of $\mathrm{Fe}^{3+}$-PEG- MNPs in BM-MSCs in vitro}

Ten milliliters of a BM-MSC suspension containing $6 \times 10^{5}$ cells per $\mathrm{mL}$ was seeded in $75 \mathrm{~cm}^{2}$ culture flasks. After $24 \mathrm{~h}$ of incubation at $37{ }^{\circ} \mathrm{C}$ in a $5 \% \mathrm{CO}_{2}$ incubator, the medium was replenished with fresh serum-free culture medium containing $\mathrm{Fe}^{3+}$-PEG-MNPs at different concentrations (0, 100, 200, 400, and $800 \mu \mathrm{g} \mathrm{mL}^{-1}$ ), and BM-MSCs were cultured for $6 \mathrm{~h}$ at $37^{\circ} \mathrm{C}$ in a $5 \% \mathrm{CO}_{2}$ incubator. Thereafter, the medium was discarded and cells were carefully washed thrice with PBS to eliminate the uninternalized $\mathrm{Fe}^{3+}$-PEG-MNPs and dead cells. After trypsinization, the cell suspension was centrifuged at room temperature (1000 rpm, $5 \mathrm{~min}$ ) and labeled stem cells were resuspended in PBS for subsequent analysis. The intracellular distribution of $\mathrm{Fe}^{3+}$-PEG-MNPs was observed via TEM and the $\mathrm{Fe}^{3+}$-PEG-MNPs labeling rate of BM-MSCs was analyzed via flow cytometry (FACS-Calibur, BD, Bergen County, New Jersey, USA).

Cell Counting Kit-8 (CCK-8) assays were performed to evaluate the cytotoxicity of $\mathrm{Fe}^{3+}$-PEG-MNPs. BM-MSCs were seeded on 96-well plates $\left(5 \times 10^{3}\right.$ cells per well), and co-incubated with $\mathrm{Fe}^{3+}$-PEG-MNPs at different concentrations $(0,12.5,25,50,100$, $200,400,800$, and $1600 \mu \mathrm{g} \mathrm{mL}{ }^{-1}$ ) for $24 \mathrm{~h}$ at $37{ }^{\circ} \mathrm{C}$ in a $5 \% \mathrm{CO}_{2}$ incubator. The medium of each well was subsequently replenished with $100 \mu \mathrm{L}$ fresh serum-free culture medium containing $10 \mu \mathrm{L}$ CCK-8 reagent (Dojindo, Lot.KR675) and incubated for $2 \mathrm{~h}$ at $37{ }^{\circ} \mathrm{C}$ in a $5 \% \mathrm{CO}_{2}$ incubator. Finally, the absorbance of each well was measured at $450 \mathrm{~nm}$ and recorded using a microplate reader (SpectraMax Plus384, MD, San Francisco, California, USA). 


\subsection{Dual-modal PA/MR imaging of BM-MSCs labeled with $\mathrm{Fe}^{3+}$-PEG-MNPs in vitro}

The $\mathrm{Fe}^{3+}$-PEG-MNP-labeled BM-MSC suspension was centrifuged (1000 rpm, $5 \mathrm{~min}$ ) at room temperature and fixed with $4 \%$ paraformaldehyde for $30 \mathrm{~min}$ at $4{ }^{\circ} \mathrm{C}$. After paraformaldehyde was removed, the fixed cells were resuspended in $100 \mu \mathrm{L}$ PBS and pipetted into 96-well PCR plates at $2 \times 10^{6}$ cells per well. Then the obtained cell suspension was imaged using MSOT, followed by 3.0-T MRI. The parameters for PAI were the same as those of the above-mentioned aqueous solution. The parameters for MRI were as follows: FOV, $90 \times 60 \mathrm{~mm}^{2}$; slice thickness, $1.5 \mathrm{~mm}$; spacing, $0.5 \mathrm{~mm}$; base resolution matrix, $256 \times 180$; TR, $500 \mathrm{~ms}$; and TE, $20 \mathrm{~ms}$.

\subsection{Dual-modal PA/MR imaging in of engrafted BM-MSCs in vivo}

Animal experiments were approved by the Institutional Animal Care and Use Committee of Shanxi Medical University (approval no. SYDL2019002) and carried out in accordance with the U.S. Guide for the Care and Use of Laboratory Animals 8th Edition 2011. ${ }^{13}$ ICR male mice (Animal Center of Shanxi Medical University, Taiyuan, China; SCXK2015-0001; 8 weeks of age, weighing 30-40 $\mathrm{g}$ each) were used herein. All injections were administered under anesthesia with sodium pentobarbital or isoflurane, and efforts were made to minimize suffering. All animals were euthanized via cervical dislocation.

Six ICR mice were randomly assigned to the following two groups ( $n=3$ /group): (1) $\mathrm{Fe}^{3+}$-PEG-MNPs injection group $\left(\mathrm{Fe}^{3+}\right.$ PEG-MNP); (2) PBS injection group (PBS). Two-hundred microliters of $8 \mathrm{mg} \mathrm{mL}{ }^{-1} \mathrm{Fe}^{3+}$-PEG-MNPs solution and PBS were injected into the tail vein of mice in the $\mathrm{Fe}^{3+}$-PEG-MNP and PBS groups, respectively. Body weights in each group were recorded and compared before injection and every $2 \mathrm{~d}$ after injection. On day 30 after injection, mice were euthanized via inhalation of an overdose of isoflurane. Thereafter, their organs including the heart, lung, liver, spleen, and kidney were dissected and assessed via HE staining.

Three mice were anesthetized via intraperitoneal administration of $1 \%$ pentobarbital sodium $\left(40 \mathrm{mg} \mathrm{kg}{ }^{-1}\right.$ ), a $50 \mu \mathrm{L}$ suspension containing $2 \times 10^{6} \mathrm{BM}$-MSCs labeled and unlabeled with $\mathrm{Fe}^{3+}$-PEG-MNPs was intramuscularly injected into the left and right thigh, respectively. PA/MR imaging of these mice were performed using MSOT, followed by 3.0-T MRI, before injection and on days 1, 7, 14, 21, 28, and 35 after BM-MSCs injection. The PA imaging parameters were as follows: temperature, $34{ }^{\circ} \mathrm{C}$; imaging wavelengths, 700, 705, 710, 730, 760, 780, 800 (used as background wavelength), 850 , and $875 \mathrm{~nm}$; frames per wavelength, 10; repetitions per slice, 1; run, 1; transverse slices thickness, $800 \mu \mathrm{m}$; step size, $0.5 \mathrm{~mm}$. The MR imaging sequence parameters were as follows: FOV, $90 \times 60 \mathrm{~mm}^{2}$; slice thickness, $1.5 \mathrm{~mm}$; spacing, $0.5 \mathrm{~mm}$; base resolution matrix, $256 \times 256$; TR, $500 \mathrm{~ms}$; and TE, $20 \mathrm{~ms}$.

\section{Results}

\subsection{Characterization of $\mathrm{Fe}^{3+}$-PEG-MNPs}

$\mathrm{Fe}^{3+}$-PEG-MNPs and PEG-MNPs displayed different UV-vis-NIR absorption spectra (Fig. 1(a)), indicating the successful synthesis of $\mathrm{Fe}^{3+}$-PEG-MNPs. As shown in (Fig. 1(b)), $\mathrm{Fe}^{3+}$-PEG-

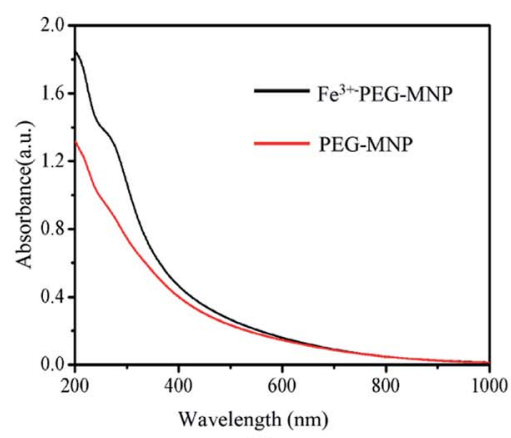

(a)

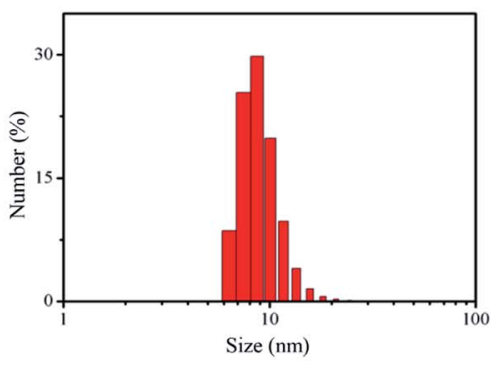

(d)

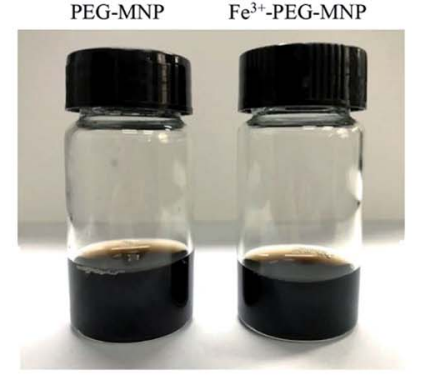

(b)

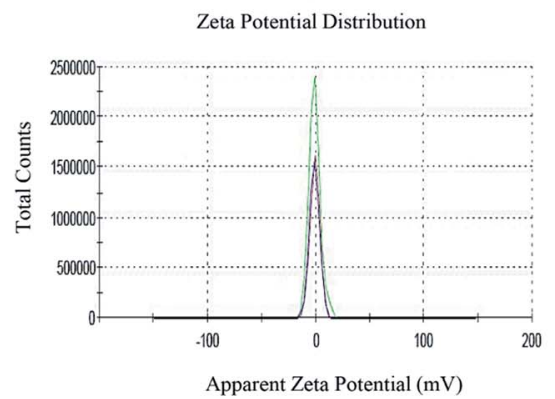

(e)

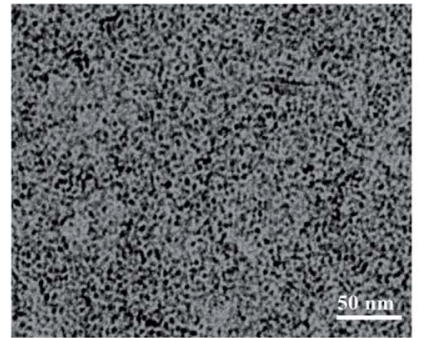

(c)

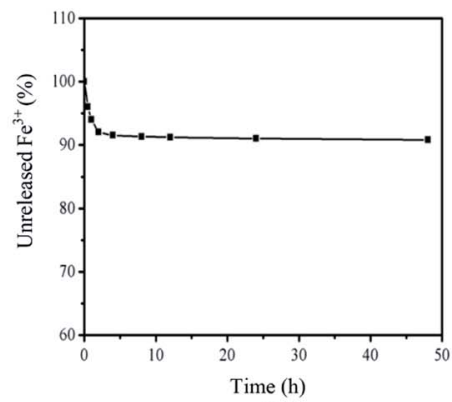

(f)

Fig. 1 Characterization of Fe ${ }^{3+}-$ PEG-MNPs: (a) UV-vis-NIR absorption spectra of aqueous solutions of Fe ${ }^{3+}-$ PEG-MNPs and PEG-MNPs; (b) aqueous solutions of PEG-MNPs and Fe $\mathrm{F}^{3+}-\mathrm{PEG}-\mathrm{MNPs}$ at $2 \mathrm{mg} \mathrm{mL}^{-1}$; (c) TEM image of Fe $\mathrm{F}^{3+}-\mathrm{PEG}-\mathrm{MNPs}$ (scale bar = $50 \mathrm{~nm}$ ); (d) size distribution of $\mathrm{Fe}^{3+}-\mathrm{PEG}-\mathrm{MNPs}$; (e) zeta potential distribution of $\mathrm{Fe}^{3+}-\mathrm{PEG}-\mathrm{MNPs}$; (f) stability analysis of $\mathrm{Fe}^{3+}-\mathrm{PEG}-\mathrm{MNPs}$ in PBS (pH $=7.4$ ). 
MNPs were highly hydrophilic. TEM images (Fig. 1(c)) of $\mathrm{Fe}^{3+}$ PEG-MNPs displayed that they had uniform size, spherical morphology, adequate monodispersion, and an even distribution. DLS analysis (Fig. 1(d) and (e)) revealed ultra-small particles with diameters of $9.2 \pm 2.2 \mathrm{~nm}$ and a neutral zeta potential of $-0.79 \pm 4.55 \mathrm{mV}$ in PBS ( $\mathrm{pH}=7.4$ ). A stability assay (Fig. 1(f)) revealed that $8 \%$ iron ions were released from $\mathrm{Fe}^{3+}$-PEG-MNPs during the initial $2 \mathrm{~h}$ and none until $48 \mathrm{~h}$, thus indicating the high stability of these particles. ICP-MS analysis revealed that the maximum quantity of $\mathrm{Fe}^{3+}$ chelated to one PEG-MNP was approximately 66 .

\subsection{Dual-modal PA/MR imaging of the aqueous solution of $\mathrm{Fe}^{3+}$-PEG-MNPs}

$T_{1}$ WI of aqueous solutions of $\mathrm{Fe}^{3+}$-PEG-MNPs and Omniscan of $0,0.0125,0.025,0.05,0.1,0.2,0.4$, and $0.8 \mathrm{mmol} \mathrm{L}^{-1}$ were obtained (Fig. 2(a)) and a $T_{1}$ WI signal intensity (SI) histogram was also plotted (Fig. 2(c)). At the same concentration, the $T_{1}$ WI SI of
$\mathrm{Fe}^{3+}$-PEG-MNPs was higher than that of Omniscan. The $r_{1}$ values represent the $T_{1}$ relaxation efficacy of $\mathrm{Fe}^{3+}$-PEG-MNPs and Omniscan, obtained by calculating the slopes of metal ions concentration with the reciprocal of the $T_{1}$ relaxation time. As shown in (Fig. 2(e)), the $r_{1}$ value of $\mathrm{Fe}^{3+}$-PEG-MNPs was higher than that of Omniscan.

The PA absorbance spectra (Fig. 2(f)) revealed that the sensitivity of MNPs was not affected upon chelation of the Fe ions but rather increased by a certain degree. PA images (Fig. 2(b)) and the signal intensity (SI) histogram (Fig. 2(d)) of the aqueous solutions of $\mathrm{Fe}^{3+}$-PEG-MNPs and PEG-MNPs of 0 , $12.5,25,50,100,200,400$, and $800 \mu \mathrm{g} \mathrm{mL}^{-1}$ also showed that the PA SI of $\mathrm{Fe}^{3+}$-PEG-MNPs was higher than that of PEG-MNPs at the same concentration.

\subsection{Successful labeling of BM-MSCs and low cytotoxicity}

As shown in TEM images (Fig. 3(a) and (b)), numerous black particles were observed in the cytoplasm of labeled rather than



(a)



(c)

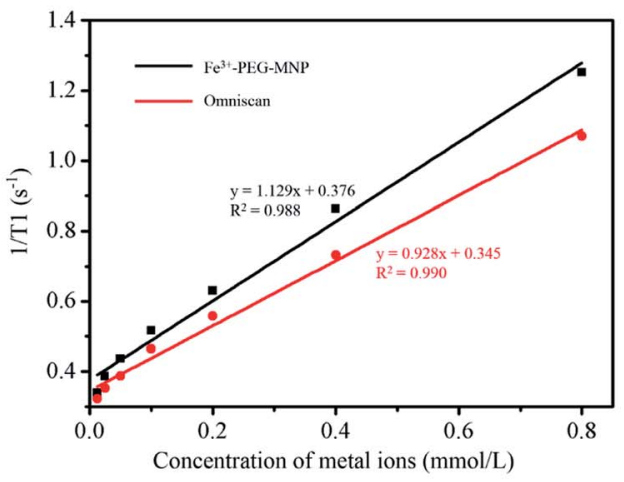

(e)

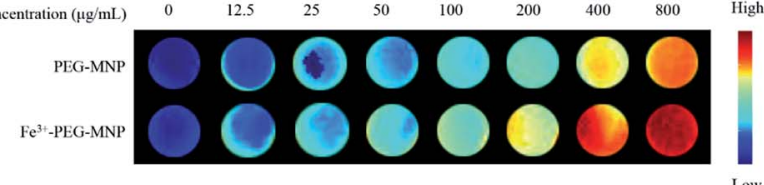

(b)

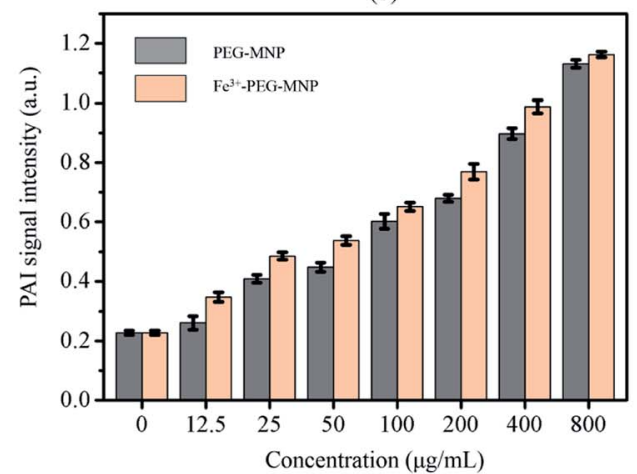

(d)



(f)

Fig. 2 MR and PA imaging Fe ${ }^{3+}$-PEG-MNPs aqueous solution: (a) MR $T_{1}$-weighted images of Fe $\mathrm{F}^{3+}-\mathrm{PEG}$-MNPs and Omniscan aqueous solutions at various concentrations of $\mathrm{Fe}^{3+}$ and $\mathrm{Gd}^{3+}\left(0,0.0125,0.025,0.05,0.1,0.2,0.4\right.$, and $0.8 \mathrm{mmol} \mathrm{L}^{-1}$ ); (b) PA images of aqueous solutions of Fe $\mathrm{P}^{3+}$ PEG-MNPs at different concentrations $\left(0,12.5,25,50,100,200,400\right.$, and $\left.800 \mu \mathrm{g} \mathrm{mL}^{-1}\right)$; signal intensity of Fe ${ }^{3+}$-PEG-MNPs at various concentrations in (c) MR and (d) PA images; (e) $T_{1}$ relaxation rates plots of Fe $\mathrm{F}^{3+}$-PEG-MNPs and Omniscan aqueous solutions based on metal ion (i.e., $\mathrm{Fe}^{3+}, \mathrm{Gd}^{3+}$ ) concentrations $\left(\mathrm{mmol} \mathrm{L}^{-1}\right)$. Each regression slope represents the $T_{1}$ relaxation efficient $\left(r_{1}\right)$; (f) photoacoustic absorbance spectra of aqueous solutions of PEG-MNPs and Fe ${ }^{3+}$-PEG-MNPs. 


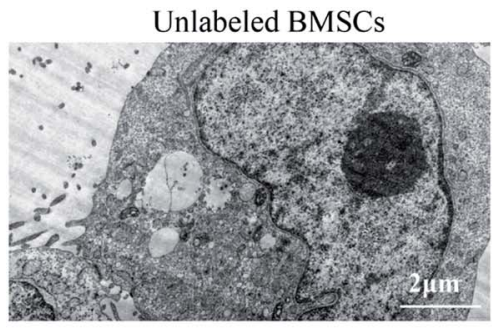

(a)

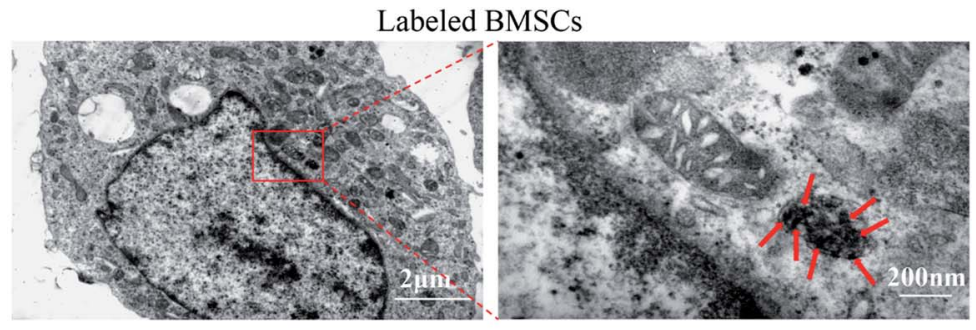

(b)
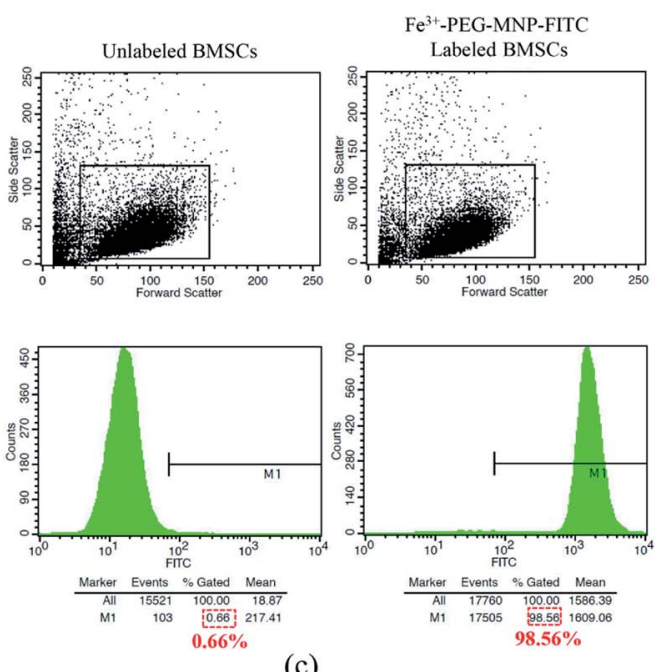

(c)

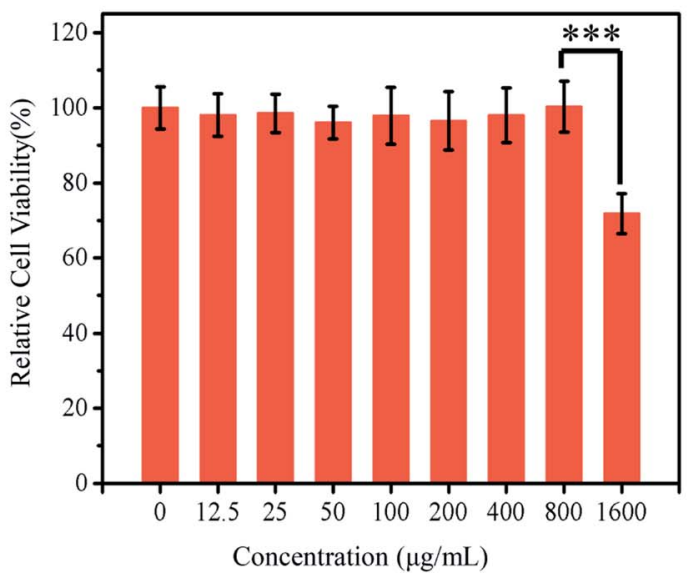

(d)

Fig. 3 ( $a$ and b) TEM images of (a) unlabeled BM-MSCs and (b) Fe ${ }^{3+}$-PEG-MNP-labeled BM-MSCs (scale bar $=2 \mu \mathrm{m}$ ). Black particles representing the internalized Fe ${ }^{3+}-$ PEG-MNPs in the cytoplasm of labeled BM-MSCs (red solid arrows in the enlarged (b)) and no black particles were observed in the organelles of unlabeled BM-MSCs; (c) the positive percentages of unlabeled and Fe ${ }^{3+}$-PEG-MNP-FITC-labeled BM-MSCs were $0.66 \%$ and $98.56 \%$, respectively; (d) results of the CCK-8 assay for BM-MSCs labeled with Fe ${ }^{3+}$-PEG-MNPs at various concentrations $(0,12.5,25,50,100$, $200,400,800$, and $1600 \mu \mathrm{g} \mathrm{mL}^{-1}$ ) in vitro. The data were analyzed using one-way ANOVA. The error bars indicate the standard deviation $(n=4$, $* * * P<0.001)$.

unlabeled BM-MSCs. Flow cytometry analysis (Fig. 3(c)) revealed that $98.56 \%$ of BM-MSCs were labeled successfully with $\mathrm{Fe}^{3+}$ PEG-MNP-FITC, indicating that $\mathrm{Fe}^{3+}$-PEG-MNPs were easily and efficiently internalized by BM-MSCs via endocytosis without the aid of a transfection agent.

On further assessing the cytotoxicity, the CCK8 assay (Fig. 3(d)) revealed that BM-MSCs exhibited higher viability of approximately $100 \%$ upon labeling with $\mathrm{Fe}^{3+}$-PEG-MNPs at $\leq 800 \mu \mathrm{g} \mathrm{mL} \mathrm{mL}^{-1}$. Although the viability of BM-MSCs labeled with $1600 \mu \mathrm{g} \mathrm{mL}^{-1} \mathrm{Fe}^{3+}$-PEG-MNPs decreased, they retained a viability of $71.8 \%$. Therefore, $\mathrm{Fe}^{3+}$-PEG-MNPs were considered safe for labeling BM-MSCs at $\leq 800 \mu \mathrm{g}$ $\mathrm{mL}^{-1}$.

\subsection{Successful dual-modal PA/MR imaging of BM-MSCs in vitro and in vivo}

To evaluate the PA and MR imaging capabilities and sensitivities of BM-MSCs labeled with $\mathrm{Fe}^{3+}$-PEG-MNPs, BM-MSCs were labeled with $\mathrm{Fe}^{3+}$-PEG-MNPs at $0,100,200,400$, and $800 \mu \mathrm{g} \mathrm{mL} \mathrm{m}^{-1}$ and subjected to PA/MR imaging. PBS constituted the control group. As shown in Fig. 4(a)-(d), the labeled BM-MSCs generated a positive signal on PAI and MR $T_{1}$ WI simultaneously and the signal intensity (SI) increased gradually with an increase in the concentration of $\mathrm{Fe}^{3+}$-PEGMNPs. BM-MSCs labeled with $800 \mu \mathrm{g} \mathrm{mL}^{-1} \mathrm{Fe}^{3+}$-PEG-MNPs exhibited the highest SI upon PA and MR $T_{1} \mathrm{WI}$ at all concentrations.

To investigate the biological safety of $\mathrm{Fe}^{3+}$-PEG-MNPs in vivo, solutions of the nanoparticles and PBS were injected into the tail vein in the $\mathrm{Fe}^{3+}$-PEG-MNP and PBS groups, respectively. No significant difference in body weight was observed between the two groups in 1 month (Fig. 5(a)), and no discernible adverse effect was observed in the primary organs of the mice in the $\mathrm{Fe}^{3+}$-PEG-MNP group, including the heart, liver, spleen, lung, and kidney (Fig. 5(b)).

PA and MR imaging of labeled BM-MSCs in vivo were further performed upon their intramuscular administration in the thigh muscle. As shown in Fig. 5(c) and (d), positive signals generated by $\mathrm{Fe}^{3+}$-PEG-MNPs-labeled BM-MSCs in the left thigh were observed, and no positive signals were generated by unlabeled BM-MSCs in the right thigh on PAI and MR $T_{1} \mathrm{WI}$; furthermore, PA and MR signals were retained for at least 35 and $28 \mathrm{~d}$, respectively. On day 35 and 28 after injection, the PA and MR signals were relatively weaker but sufficient to facilitate monitoring of engrafted BM-MSCs. 


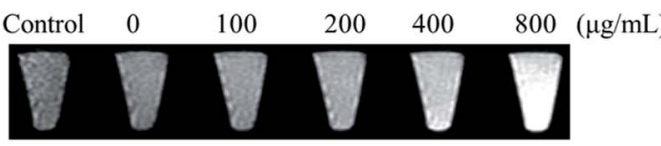

(a)

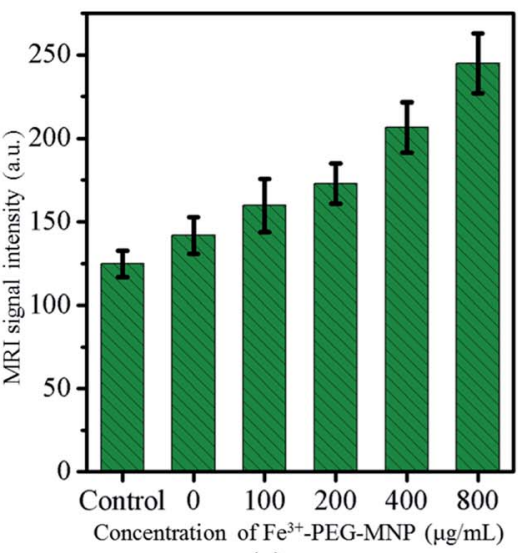

(c)

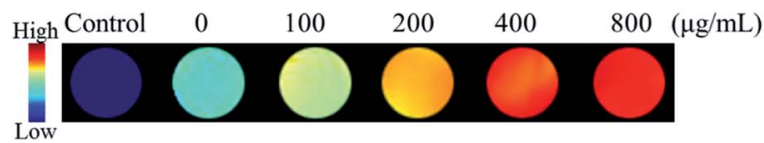

(b)

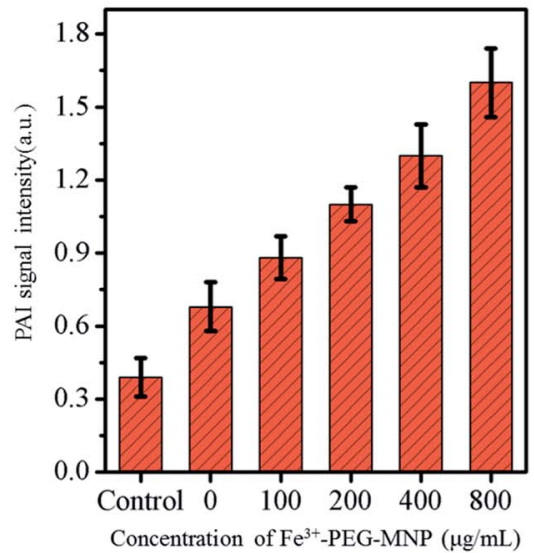

(d)

Fig. 4 In vitro MR and PA images of BM-MSCs labeled with Fe ${ }^{3+}$-PEG-MNPs: (a) MR $T_{1}$-weighted images and (b) PA images of BM-MSCs labeled with $\mathrm{Fe}^{3+}$-PEG-MNPs at various concentrations $\left(0,100,200,400\right.$, and $800 \mu \mathrm{g} \mathrm{mL}^{-1}$ ). PBS was used as the control; (c) MR and (d) PA signal intensity of BM-MSCs labeled with Fe ${ }^{3+}$-PEG-MNPs at various concentrations $\left(0,100,200,400\right.$, and $\left.800 \mu \mathrm{gL}^{-1}\right)$.

(a)

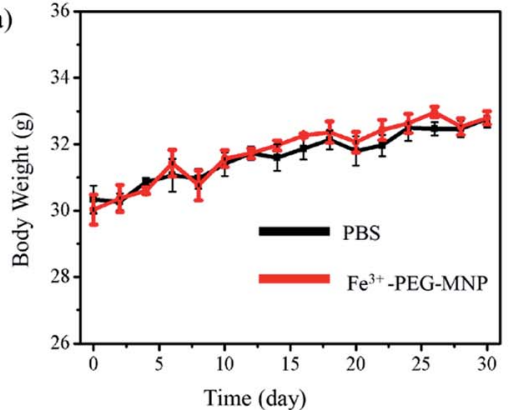

(b)



(c)

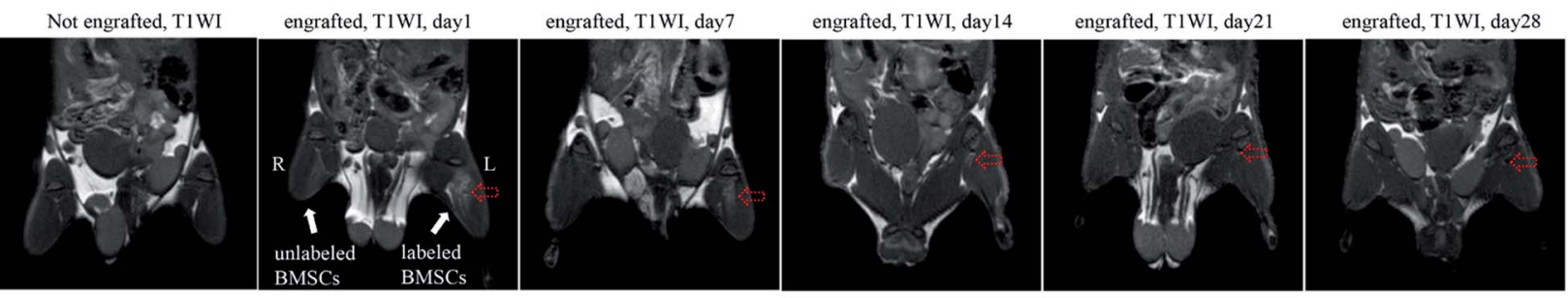

(d)
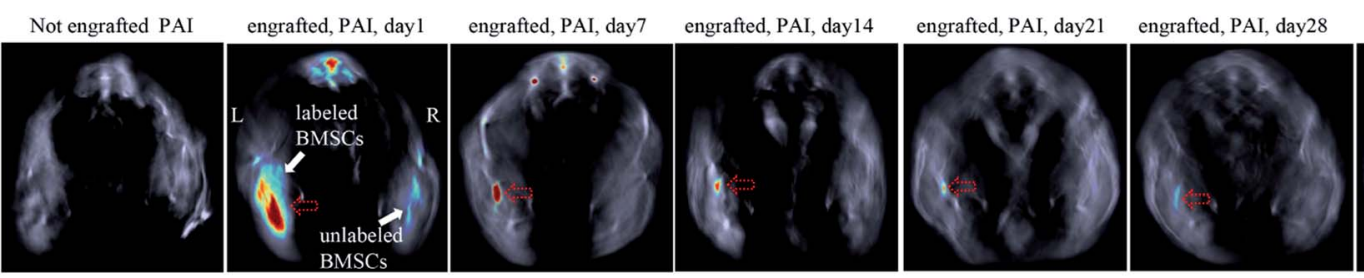

engrafted, PAI, day35 High

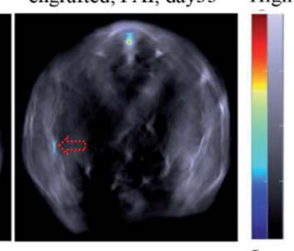

Fig. 5 (a) Changes in the body weight of ICR mice from the Fe ${ }^{3+}-\mathrm{PEG}-\mathrm{MNP}$ and PBS groups in $30 \mathrm{~d}$ after Fe ${ }^{3+}-\mathrm{PEG}-\mathrm{MNP}$ and PBS injection; (b) $\mathrm{HE}$ staining of the primary organs of the ICR mice from the Fe ${ }^{3+}-\mathrm{PEG}-\mathrm{MNP}$ and PBS groups on day 30 after Fe $\mathrm{F}^{3+}-\mathrm{PEG}-\mathrm{MNP}$ and PBS injection (scale bar $=200 \mu \mathrm{m}$ ); (c) coronal $T_{1} \mathrm{WI}$ and (d) transverse PAI of the ICR mice before and after intramuscular injection of BM-MSCs into the thigh muscles of mice. The engrafted BM-MSCs labeled with $\mathrm{Fe}^{3+}-\mathrm{PEG}-\mathrm{MNPs}$ are indicated by the red hollow dashed arrows. $\mathrm{L}$ and $\mathrm{R}$ represent the left and right thigh, respectively. 


\section{Discussion}

Stem cell therapy (especially with BM-MSCs) has beneficial effects and is potentially applicable for numerous diseases and disorders. ${ }^{14-16}$ In vivo cell imaging is essential to ensure appropriate delivery of stem cells, monitoring of the homing, survival, and distribution of stem cells, and understanding the mechanism underlying stem cell-mediated functional recovery. ${ }^{17-19}$ Various imaging methods have been widely used for cell tracking, primarily including optical imaging, MRI, PET, and PAI. However, these imaging methods have intrinsic and inevitable limitations; hence, none of them satisfy all clinical or experimental requirements of stem cell tracking. To circumvent these limitations and achieve synergistic advantages, dualmodal or multimodal imaging of cells has been proposed in this study as an alternative and promising strategy for cells tracking. ${ }^{17}$

Currently, MRI is not only a prevalent tool for tracking noninvasive cells in vivo but also a common diagnostic tool for medical imaging in the clinical setting. ${ }^{\mathbf{8 , 2 0 , 2 1}}$ MRI has highspatial resolution (up to $100 \mu \mathrm{m}$ ) and is capable of long-term cell tracking (up to 6 weeks). ${ }^{22,23}$ However, the clinical application of stem cell imaging is limited by its intrinsic deficiencies, including relative low sensitivity, long imaging duration, and the contraindications after the implantation of metal devices. ${ }^{\mathbf{8 2 4}}$ To circumvent these limitations and improve cell-imaging modalities, this study used a dual-modal imaging strategy involving photoacoustic and magnetic resonance imaging for monitoring stem cells in vivo. PAI is an excellent and rapidly evolving biomedical imaging method. Briefly, a ultrasound wave can be detected by US detector, which is generated by an instant thermoelastic expansion of tissue after photon absorption by tissues. ${ }^{25} \mathrm{PAI}$ is a promising stem cell imaging modality with high sensitivity of optical imaging, outstanding resolution of micrometers equivalent to the size of an organelle and a favorable tissue penetration depth of several centimeters. $^{\text {10,26,27 }}$ Furthermore, dual-modal PA/MR imaging compensates for the limitations of each individual modality; hence, the sensitivity and specificity of cell tracking in vivo can be improved.

Successful and effective cell tracking in vivo requires cell tracers with outstanding properties, including high stability, effective cell labeling, low cytotoxicity, high biosafety, excellent imaging capability. ${ }^{28}$ Nanoparticles have been widely used as stem cell trackers in vivo. In this study, we designed and synthesized novel dual-modal PA/MR nanoparticles $\left(\mathrm{Fe}^{3+}\right.$-PEGMNPs) containing melanin and iron ions.

Melanin is a ubiquitous pigment in nature and is widespread in the tissues of many organisms, including human skin, mucous membranes, retina, cerebral pia mater, and ovaries. ${ }^{\mathbf{1 1 , 2 9 , 3 0}}$ In addition, it actively chelates various metal ions such as ${ }^{64} \mathrm{Cu}^{2+}, \mathrm{Fe}^{3+}, \mathrm{Gd}^{3+}$, and $\mathrm{Mn}^{2+} \cdot{ }^{11}$ Iron is an essential trace element for organismal growth. In the human body, iron is stored as ferritin in the liver, spleen, small intestine mucosa, bone marrow, and other organs or tissues and is primarily excreted through the intestines. Therefore, the novel nanoparticle ( $\mathrm{Fe}^{3+}$-PEG-MNP) synthesized herein on the basis of these two biomaterials is expected to display adequate biological safety. As expected, this study shows that the viability of labeled BM-MSCs can be maintained at high levels even at 1600 $\mu \mathrm{g} \mathrm{mL} \mathrm{m}^{-1}$ of $\mathrm{Fe}^{3+}$-PEG-MNPs (Fig. 3(d)). Moreover, the body weights of the mice were not affected, and no discernible adverse effects were observed in the heart, lung, liver, spleen, and kidney after the injection of $\mathrm{Fe}^{3+}$-PEG-MNPs (Fig. 5(a) and (b)). These results indicate the high biological safety of $\mathrm{Fe}^{3+}$ PEG-MNPs.

PEG-MNPs were previously used as a nanoplatform for multimodal imaging and imaging-guided nanoscale systems for drug delivery. ${ }^{11,31}$ Herein, $\mathrm{Fe}^{3+}$-PEG-MNPs were easily obtained by stirring at $37^{\circ} \mathrm{C}$ for $1 \mathrm{~h}$. BM-MSCs were easily and efficiently labeled with $\mathrm{Fe}^{3+}$-PEG-MNPs via co-incubation without the aid of any transfection agent (Fig. 3(a)-(c)) owing to the ultra-small size of approximately $9.2 \mathrm{~nm}$ and surface neutral charge of $\mathrm{Fe}^{3+}$ PEG-MNPs (Fig. 1(d) and (e)). After redissolution in PBS, only approximately $8 \%$ of $\mathrm{Fe}^{3+}$ was released from $\mathrm{Fe}^{3+}$-PEG-MNPs during the initial $2 \mathrm{~h}$ and the release ratio of $\mathrm{Fe}^{3+}$ was subsequently maintained at this relatively low level, thus indicating the high stability of $\mathrm{Fe}^{3+}$-PEG-MNPs (Fig. 1(f)). As melanin and $\mathrm{Fe}^{3+}$ could yielded PA and MR signals, respectively, ${ }^{11,32-34}$ both PAI and MR $T_{1}$ WI of BM-MSCs were successfully accomplished using $\mathrm{Fe}^{3+}$-PEG-MNPs in vitro and in vivo (Fig. 4(a) and (b) and 5(c) and (d)). In particular, approximately 1 month after cell transplantation, the PA and MR SI of BM-MSCs labeled with $\mathrm{Fe}^{3+}$-PEG-MNPs in vivo were maintained at a relatively stable level to adequately facilitate the monitoring of engrafted cells (Fig. 5(c) and (d)). In the future studies, $\mathrm{Fe}^{3+}$-PEG-MNPs will be used for monitoring BM-MSCs in animal model of human disease to understand some repair mechanisms underlying BMMSC-mediated.

\section{Conclusions}

This study describes a novel dual-modal PA/MR nanoparticle $\left(\mathrm{Fe}^{3+}\right.$-PEG-MNP) probe based on natural biomaterials for monitoring engrafted BM-MSCs. Briefly, $\mathrm{Fe}^{3+}$-PEG-MNPs are advantageous for tracking engrafted BM-MSCs owing to their simple synthesis, high stability and hydrophilicity, ease of labeling cells and low cytotoxicity, high biological safety, excellent capability of dual-modal PA/MR imaging, high sensitivity and long-term monitoring in vitro and in vivo. Based on these desired properties, $\mathrm{Fe}^{3+}$-PEG-MNPs are an ideal dualmodal PA/MR nanoparticle probe for future clinical SCT, thus contributing to advancements in basic studies using stem cells.

\section{Author contributions}

Hua Zhang conceived and designed the experiment, prepared the samples, performed the synthesis experiments and revised the manuscript. Zhen-Jun Wang and Ling-Jie Wang analyzed the data and wrote the manuscript. Ting-Ting Li performed the characterization of $\mathrm{Fe}^{3+}$-PEG-MNP. Sheng He contributed to the extracting and culturing BM-MSCs. Li-Ping Li, Xiao-Yan Li and Shi-Jie Liu performed the experiments in vitro and in vivo. Jian- 
Ding Li contributed to the experimental instruments. Si-Jin Li and Rui-Ping Zhang supervised the entire project and integrated the different parts of the work together. All authors approved the final manuscript.

\section{Conflicts of interest}

The authors declare no conflicts of interest.

\section{Acknowledgements}

This work was financially supported by the National Natural Science Foundation of China (no. 81571747 and 81771907), Key Research and Development Project of Shanxi Province (no. 201703D321015-3, 201803D31004 and 201803D31109), Engineering Technology Research Center of Shanxi Province (no. 201805D121008), Science and Technology Innovation Team Project of Shanxi Province (no. 201705D131026), Scientific and Technological Achievements Transformation Project of Shanxi Province (no. 201704D131006), Laboratory Construction Project of Shanxi Province, and the Projects for Local Science and Technology Development Guided by the Central Committee (no. YDZX20191400002537).

\section{References}

1 G. I. Im, Eur. Cells Mater., 2017, 33, 183-196.

2 B. Lv, F. Li, J. Han, J. Fang, L. Xu, C. Sun, T. Hua, Z. Zhang, Z. Feng and X. Jiang, Front. Mol. Neurosci., 2017, 10, 80.

3 D. Shi, J. Zhang, Q. Zhou, J. Xin, J. Jiang, L. Jiang, T. Wu, J. Li, W. Ding, J. Li, S. Sun, J. Li, N. Zhou, L. Zhang, L. Jin, S. Hao, P. Chen, H. Cao, M. Li, L. Li, X. Chen and J. Li, Gut, 2017, 66, 955-964.

4 X. Yuan, X. Wang, C. Chen, J. Zhou and M. Han, Stem Cell Res. Ther., 2017, 8, 146.

5 W. Liu, Y. Wang, F. Gong, Y. Rong, Y. Luo, P. Tang, Z. Zhou, Z. Zhou, T. Xu, T. Jiang, S. Yang, G. Yin, J. Chen, J. Fan and W. Cai, J. Neurotrauma, 2019, 36, 469-484.

6 V. Jeevanantham, M. Butler, A. Saad, A. Abdel-Latif, E. K. Zuba-Surma and B. Dawn, Circulation, 2012, 126, 551568.

7 T. Schroeder, Nature, 2008, 453, 345-351.

8 S. J. Zhang and J. C. Wu, J. Nucl. Med., 2007, 48, 1916-1919.

9 J. E. Lemaster, C. Fang, T. Kim, A. Hariri and J. V. Jokerst, ACS Appl. Nano Mater., 2018, 1, 1321-1331.

10 L. V. Wang and S. Hu, Science, 2012, 335, 1458-1462.

11 Q. Fan, K. Cheng, X. Hu, X. Ma, R. Zhang, M. Yang, X. Lu, L. Xing, W. Huang, S. S. Gambhir and Z. Cheng, J. Am. Chem. Soc., 2014, 136, 15185-15194.

12 W. W. Cai, L. J. Wang, S. J. Li, X. P. Zhang, T. T. Li, Y. H. Wang, X. Yang, J. Xie, J. D. Li and S. J. Liu, J. Biomed. Mater. Res., Part A, 2017, 105, 131-137.
13 National Research Council, Guide for the care and use of laboratory animals, National Academies Press, Washington, DC, 8th edn, 2011.

14 R. C.-J. Chiu, Heart Failure Rev., 2003, 8, 247-251.

15 X. Chenjie, M. N. David, J. A. Ankrum, M. Mads Emil, J. A. Phillips, R. Isaac, G. R. Wojtkiewicz, J. Vikram, K. Jens Roat and Z. Weian, Nano Lett., 2012, 12, 4131-4139.

16 R. C.-J. Chiu, Heart Failure Rev., 2003, 8, 247-251.

17 N. Sugiyama, A. Y. Sonay, R. Tussiwand, B. E. Cohen and P. Pantazis, Small, 2018, 14, 1703386.

18 J. V. Jokerst, M. Thangaraj, P. J. Kempen, R. Sinclair and S. S. Gambhir, ACS Nano, 2012, 6, 5920-5930.

19 J. E. Lemaster, F. Chen, T. Kim, A. Hariri and J. V. Jokerst, ACS Appl. Nano Mater., 2018, 1, 1321-1331.

20 S. J. Liu, L. J. Wang, Y. Qiao, H. Zhang, L. P. Li, J. H. Sun, S. He, W. Xu, X. Yang, W. W. Cai, J. D. Li, B. Q. Wang and R. P. Zhang, Int. J. Nanomed., 2018, 13, 1749-1759.

21 M. E. Kupfer and B. M. Ogle, Biotechnol. J., 2015, 10, 15151528.

22 K. Daehong, H. Kwan Soo and S. Jihwan, Mol. Cells, 2007, 23, 132.

23 C. Xu, D. Miranda-Nieves, J. A. Ankrum, M. E. Matthiesen, J. A. Phillips, I. Roes, G. R. Wojtkiewicz, V. Juneja, J. R. Kultima, W. Zhao, P. K. Vemula, C. P. Lin, M. Nahrendorf and J. M. Karp, Nano Lett., 2012, 12, 41314139.

24 J. E. Lemaster, Z. Wang, A. Hariri, F. Chen, Z. Hu, Y. Huang, C. V. Barback, R. Cochran, N. C. Gianneschi and J. V. Jokerst, Chem. Mater., 2018, 31, 251-259.

25 Q. Fu, R. Zhu, J. Song, H. Yang and X. Chen, Adv. Mater., 2019, 31, e1805875.

26 X. Qin, H. Chen, H. Yang, H. Wu, X. Zhao, H. Wang, T. Chour, E. Neofytou, D. Ding, H. Daldrup-Link, S. C. Heilshorn, K. Li and J. C. Wu, Adv. Funct. Mater., 2018, 28, 1704939.

27 J. Weber, P. C. Beard and S. E. Bohndiek, Nat. Methods, 2016, 13, 639-650.

28 J. Wang and J. V. Jokerst, Stem Cells Int., 2016, 2016, 9240652. 29 P. A. Riley, Int. J. Biochem. Cell Biol., 1997, 29, 1235-1239.

30 D. L. Longo, R. Stefania, C. Callari, F. De Rose, R. Rolle, L. Conti, L. Consolino, F. Arena and S. Aime, Adv. Healthcare Mater., 2017, 6, 1600550.

31 R. Zhang, Q. Fan, M. Yang, K. Cheng, X. Lu, L. Zhang, W. Huang and Z. Cheng, Adv. Mater., 2015, 27, 5063-5069.

32 K. Y. Ju, J. Kang, J. Pyo, J. Lim, J. H. Chang and J. K. Lee, Nanoscale, 2016, 8, 14448-14456.

33 H. T. Ta, Z. Li, C. E. Hagemeyer, G. Cowin, S. Zhang, J. Palasubramaniam, K. Alt, X. Wang, K. Peter and A. K. Whittaker, Biomaterials, 2017, 134, 31-42.

34 N. Kuźnik, M. M. Tomczyk, M. Wyskocka, Ł. Przypis, A. P. Herman, R. Jędrysiak, K. K. Koziol and S. Boncel, Int. J. Nanomed., 2015, 10, 3581. 